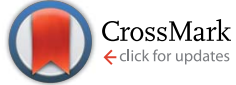

Cite this: J. Mater. Chem. A, 2014, 2 , 14807

Received 5th June 2014 Accepted 14th July 2014

DOI: $10.1039 / c 4 t a 02831 \mathrm{~g}$

www.rsc.org/MaterialsA

\section{Enhanced catalytic application of Auapolyphenol- metal nanocomposites synthesized by a facile and green method $\dagger$}

\author{
Tao Zeng, ${ }^{a}$ Xiaole Zhang, ${ }^{\text {ab }}$ Yuanyuan Guo, ${ }^{\text {ac }}$ Hongyun Niu ${ }^{a}$ and Yaqi Cai ${ }^{\star a}$
}

Development of novel nanostructure gold-based catalysts with high performance and low cost is of a great scientific interest. Here, a new controllable coordination coating was facilely integrated onto gold nanoparticles (Au NPs) using low-cost natural polyphenols and ferric ions. Heat treatment, organic solvents, special instruments, or additive chemicals were not involved in the whole synthetic procedure. Due to the presence of the unique polyphenol- $\mathrm{Fe}^{3+}$ shell, the as-prepared catalyst showed an improved catalytic performance, $\mathrm{pH}$-responsive character and good stability towards the reduction of 4-NP.

Noble metal NPs have received enormous interest in a wide variety of applications, especially in heterogeneous catalysis owing to their large specific surface areas and increased number of exposed metal atoms, which greatly change their catalytic and physicochemical properties. ${ }^{1,2}$ Among them, Au NPs have been proved to exhibit extraordinary performance in a number of oxidation and reduction reactions. ${ }^{3-5}$ However, Au NPs in catalytic reactions often show a strong tendency to aggregate because of their high surface energies, leading to the loss of their initial activities. ${ }^{6}$ Hence, core-shell architectures with a noble metal core preserved by an outer shell have recently attracted a great deal of attention for catalysis due to the following structural advantages: (a) the shell can hinder the aggregation of neighboring cores ${ }^{7,8}$ and (b) remarkable catalytic activity may be achieved due to the unique identity of the shell or the interaction of the shell and the core. ${ }^{9,10}$ Over the years, a series of intriguing materials have been put into the design of such core-shell nanocatalyst systems with some specific merits. ${ }^{11,12}$ To name a few, wrapping Au NPs within mesoporous

\footnotetext{
${ }^{a}$ State Key Laboratory of Environmental Chemistry and Ecotoxicology, Research Center for Eco-Environmental Sciences, Chinese Academy of Sciences, P.O. Box 2871, Beijing 100085, China. E-mail: caiyaqi@rcees.ac.cn; Tel: +86-10-6284-9182

${ }^{b}$ College of life science, Hebei United University, Tangshan, Hebei, 06300o, China

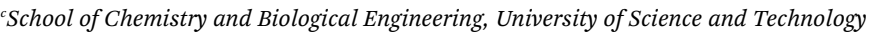
Beijing, Beijing, 100083, China

$\dagger$ Electronic supplementary information (ESI) available: Experimental details and additional figures. See DOI: 10.1039/c4ta02831g
}

silica shells to endow them with high activity and outstanding stability even under harsh conditions; ${ }^{13}$ coating metal-organic frameworks onto the surface of Au NPs to enhance the catalytic activity through a synergistic effect; ${ }^{14}$ encapsulating Au NPs within stimuli-responsive polymers to tune the mass transport of reactants and thus modulate the catalytic process. ${ }^{15}$ However, the above-mentioned synthetic procedures often suffer from sophisticated and time-consuming steps and requirement of various toxic reagents, which may be impediments for some practical applications. Accordingly, a concise, facile and environmentally friendly approach should be developed to obtain a novel core-shell Au catalytic system with distinct performance.

Polyphenols (PPs), which are commonly distributed in plant tissues to serve diverse biological roles, represent a wide range of physicochemical natures and thereby give rise to broad chemical versatility in industrial, pharmacological, biomedical, and food additive applications. ${ }^{16,17}$ The dominant constituents for virtually all PPs are catechol and galloyl, which are known to possess strong metal chelation ability and materials surface binding affinity. ${ }^{\mathbf{1 8 , 1 9}}$ Recently, researchers found that catechol units and catechol ferric ion complexes in adhesive proteins greatly account for the excellent adhesive ability of marine mussels. ${ }^{20}$ Inspired by these findings, PPs would thus display great potential in the context of surface modification based on the PPs-metal coordination. Compared with the recent high profile natural polydopamine coating, PPs-metal coating has a lower cost in terms of reagent expense. ${ }^{16}$ Moreover, the polymerlike supramolecular structure of the PPs-metal coating has an additional advantage over other inorganic layers in that it may present stimuli responsiveness, which is very useful for external manipulation in catalysis. ${ }^{21}$ To date, there has been only limited information on the PPs-metal coatings, and studies on the systematic synthesis of such coating-based core-shell nanostructures as well as their performance in the catalytic field remain unexplored.

Herein, we report a simple, fast and green approach to fabricate a novel core-shell catalyst system by applying one-step assembly of PPs-metal coordination complexes onto Au NP 
surfaces. The preparation procedure of this catalyst is schematically illustrated in Fig. 1 [here tannic acid (TA) was chosen as a representative PP]. First, Au NPs were synthesized at room temperature by taking advantage of the good reducing ability of TA toward $\mathrm{HAuCl}_{4}$ so that additional toxic reductants were not needed, which has been confirmed by previous studies. ${ }^{22-24}$ Afterwards, a supramolecular shell was generated onto the surface of the Au NPs by mixing TA and $\mathrm{Fe}^{3+}$ within the asprepared Au NPs solution. In this step, TA acted as an organic ligand, while $\mathrm{Fe}^{3+}$ was used as an inorganic cross-linker. The galloyl groups from several TA molecules can chelate a $\mathrm{Fe}^{3+}$ ion to form a stable octahedral complex, ${ }^{25}$ resulting in the construction of a cross-linked $\mathrm{TA}-\mathrm{Fe}^{3+}$ supramolecular shell. Coupled with the perfect surface binding affinity of TA, the shell was therefore easily coated onto the surface of the Au NPs. It is noteworthy that the overall synthetic procedure could be conducted rapidly in aqueous solution at ambient temperature with TA and $\mathrm{FeCl}_{3}$ as the only involved reagents. Thus, heat treatment, organic solvents, special instruments, and additive chemicals are not necessary to fabricate such Au@PPs-metal complex nanocatalysts, which greatly reduces the cost and prevents possible contamination. Moreover, this Au@PPs-metal catalyst shows $\mathrm{pH}$-responsive character and enhanced catalytic activity for the reduction of 4-nitrophenol (4-NP).

After the addition of TA into $\mathrm{HAuCl}_{4}$ solution with 1 min of mixing, the color of mixture turned to claret-red, indicating the generation of Au NPs. From the TEM image (Fig. S1, see ESI $\dagger$ ), the as-prepared Au NPs are nearly spherical with a uniform size of $\sim 35 \mathrm{~nm}$ in diameter. Upon another rapid mixing of $\mathrm{TA}, \mathrm{Fe}^{3+}$, and $\mathrm{Au}$ NPs, core-shell structures with deep-contrast cores and light-contrast layers were formed and revealed by the TEM observations (Fig. 2a-c). The bright parts in the high angular annular dark field STEM (HAADF-STEM) can be regarded as the $\mathrm{Au}$ cores (Fig 2d). Furthermore, integration of the TA-Fe ${ }^{3+}$ shell onto the surface of the Au cores decreased the zeta potential from $-10.5 \mathrm{mV}$ to $-\mathbf{1 8 . 3} \mathrm{mV}$ due to the presence of acidic galloyl groups in TA. Interestingly, the shell thickness can be readily tuned by simply repeating the coating procedure through an analogous layer-by-layer (LbL) assembly; the shell thickness

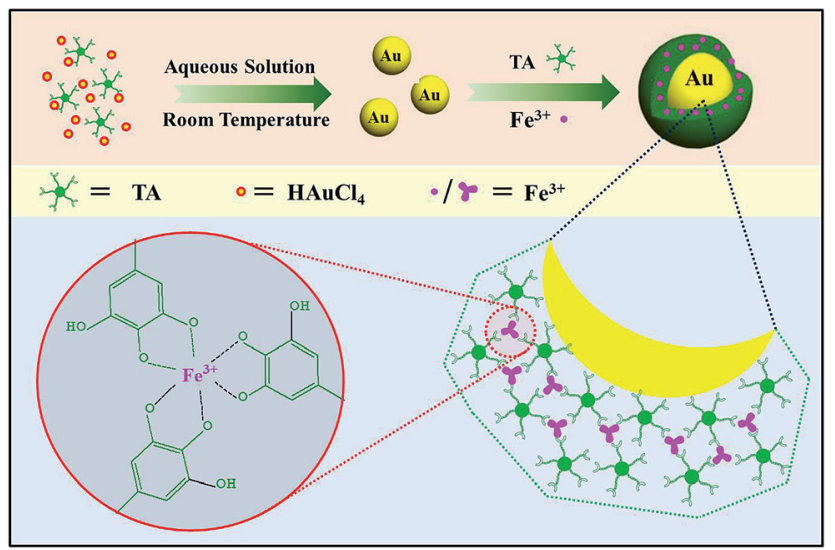

Fig. 1 Schematic illustration of the fabrication of a core-shell Au@TA-Fe nanocatalyst.

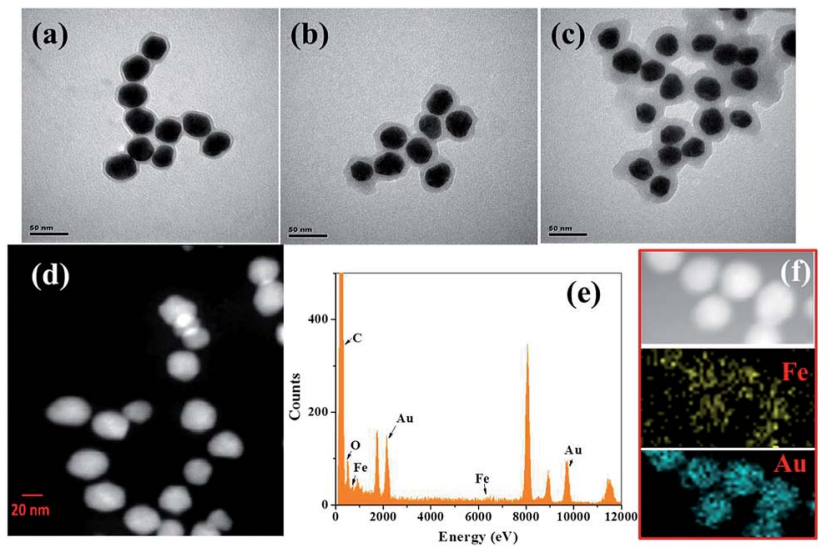

Fig. 2 TEM images of AuQTA-Fe composites with one (a), two (b), and three assembly cycles (c). HAADF-STEM image (c), EDX spectra (e), and elemental mapping (f) of Au(TA-Fe composites.

increased from $2.5 \mathrm{~nm}$ to $10.1 \mathrm{~nm}$ and $14.8 \mathrm{~nm}$ when the coating cycle was repeated with 1,2 , and 3 rounds (shown in Fig. 2a-c). Besides, as the repeat time increased, the increase of the TA-Fe ${ }^{3+}$ shell thickness could be also visually witnessed by the gradual deepening of the solution color (the inset in Fig. S2 $\dagger$ ). This phenomenon was further supported by the redshift of the peak plasmon absorption in the UV-vis tests (Fig. S2 $\dagger$ ), which is correlated with a change in the local dielectric constant or the slight fusion of Au NPs as a result of the formation of TA- $\mathrm{Fe}^{3+}$ complexes onto their surfaces. ${ }^{26}$ The energy dispersive X-ray (EDX) spectrum in Fig. 2e disclosed the elemental compositions of the nanocomposites and the observed $\mathrm{C}, \mathrm{O}, \mathrm{Fe}$, and $\mathrm{Au}$ elements ensure the involvement of $\mathrm{TA}, \mathrm{Fe}^{3+}$, and Au NPs in them. A more detailed elemental distribution was gained from EDX mapping analyses. The Au signal intensively dispersed the core zones, while the Fe signal was relatively extensively detected all over the materials surface, suggesting the integration of the core-shell Au@TA-Fe systems. Based on the above results, other PPs, such as epigallocatechin gallate (EGCG) and gallic acid (GA) were also used to prepare such Au@PPs-metal catalysts, and the TEM results are shown in

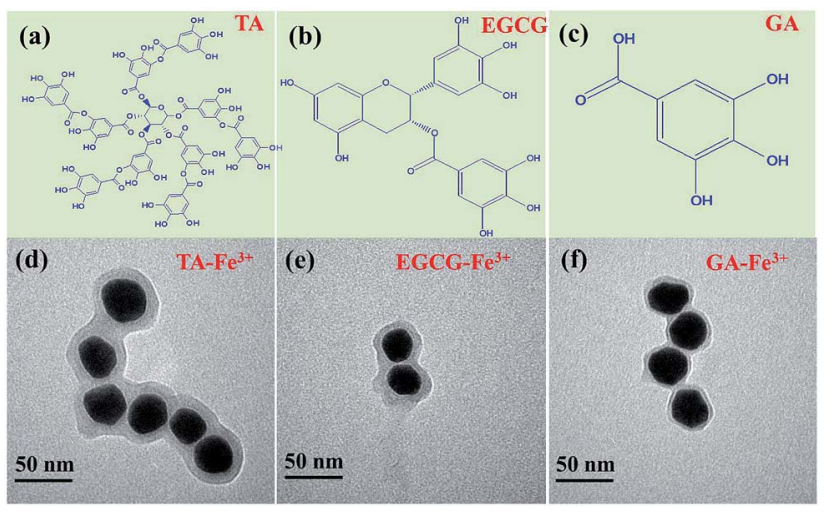

Fig. 3 Chemical structures of the selected PPs: TA (a), EGCG (b), and GA (c). TEM images of the formation of core-shell AuCTA-Fe (d), Au@EGCG-Fe (e), and Au@GA-Fe (f). 
Fig. 3. It can be seen that the core-shell structures were successfully constructed and the shell thicknesses of Au@TAFe, Au@EGCG-Fe, and Au@GA-Fe after two repeats of the cycle were $10.1 \mathrm{~nm}, 8.1 \mathrm{~nm}$, and $4.0 \mathrm{~nm}$, respectively. The disparity in shell thickness may be ascribed to the different molecular weight of various PPs. ${ }^{27}$ This demonstrated the general applicability of this method in the engineering of Au-based coreshell catalysts.

To further confirm the surface chemical information of the nanocomposites, XPS analysis was performed. Fig. 4a displays the survey scan spectrum of Au@TA-Fe, in which C 1s, O 1s, Fe $2 \mathrm{p}$, and $\mathrm{Au} 4 \mathrm{f}$ core-levels clearly exist, and this is consistent with the EDX results. From Fig. 4b, the $\mathrm{C}$ 1s core-level photoelectron spectrum can be curved into three peak components located at $284.7 \mathrm{eV}, 286.3 \mathrm{eV}$, and $288.5 \mathrm{eV}$, which are assigned to $\mathrm{C}-\mathrm{C}$, $\mathrm{C}-\mathrm{O}$, and $\mathrm{C}=\mathrm{O}$ or $\mathrm{O}-\mathrm{C}=\mathrm{O}$ groups from $\mathrm{TA}$, respectively. ${ }^{28}$ The Fe $2 \mathrm{p}$ signal range from $710.6 \mathrm{eV}$ to $724.2 \mathrm{eV}$ showed a peak separation of $\sim 12-14 \mathrm{eV}$ (Fig. 4c), implying the presence of ferric species. ${ }^{29}$ In addition, the peak appearing at $531.2 \mathrm{eV}$ in the split $\mathrm{O}$ 1s spectrum (Fig. $\mathrm{S} 3 \dagger$ ) can be attributed to $\mathrm{Fe}-\mathrm{O}$ species arising from the coordination between TA and $\mathrm{Fe}^{3+} \cdot{ }^{30}$ The $\mathrm{Au} 4 \mathrm{f}$ peaks at 84.3 and $87.9 \mathrm{eV}$ are geared to the $\mathrm{Au} 4 \mathrm{f}_{7 / 2}$ and $\mathrm{Au} 4 \mathrm{f}_{5 / 2}$ of $\mathrm{Au}^{0}$, respectively (Fig. S4†), suggesting the existence of $\mathrm{Au}$ NPs. ${ }^{31}$

The catalytic activity of Au@TA-Fe composites was checked by choosing the reduction of 4-NP to 4-aminophenol (4-AP) with $\mathrm{NaBH}_{4}$ as a model reaction, which was monitored by UV-vis spectra as a function of time. As shown in Fig. 5, the peak at $400 \mathrm{~nm}$ attributed to 4-nitrophenate came into being immediately after the addition of $\mathrm{NaBH}_{4}$ and gradually decreased with time after the introduction of the Au@TA-Fe catalyst. At the same time, a new peak at $312 \mathrm{~nm}$ appeared, indicating the production of 4-AP. The reaction was completed within several minutes and was accompanied by the discolourization of the initial yellow solution. The reaction did not proceed in the absence of Au@TA-Fe catalyst, implying its necessity in the

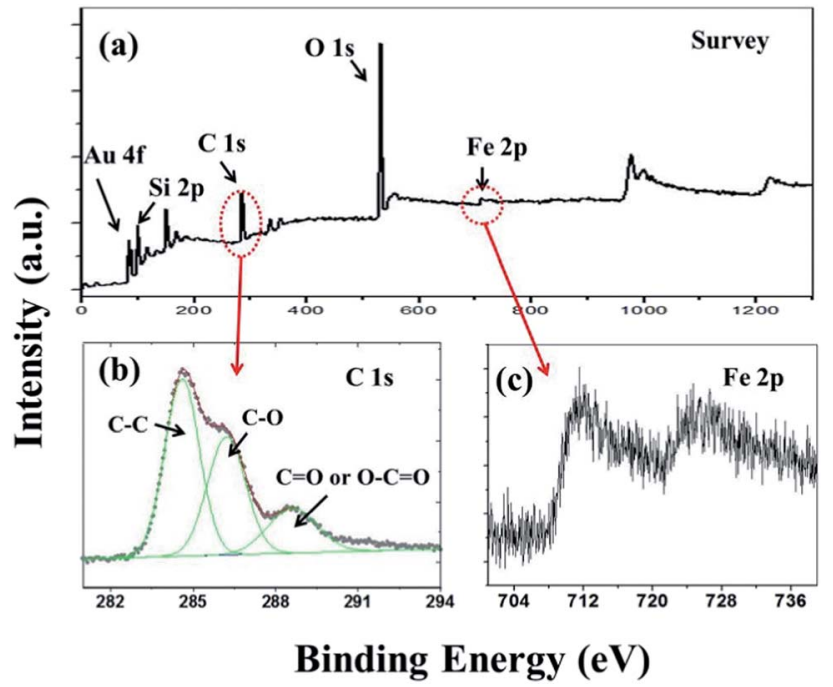

Fig. 4 XPS survey spectrum (a), C 1s core-level spectrum (b), and Fe $2 p$ core-level spectrum (c) of Au@TA-Fe composites.

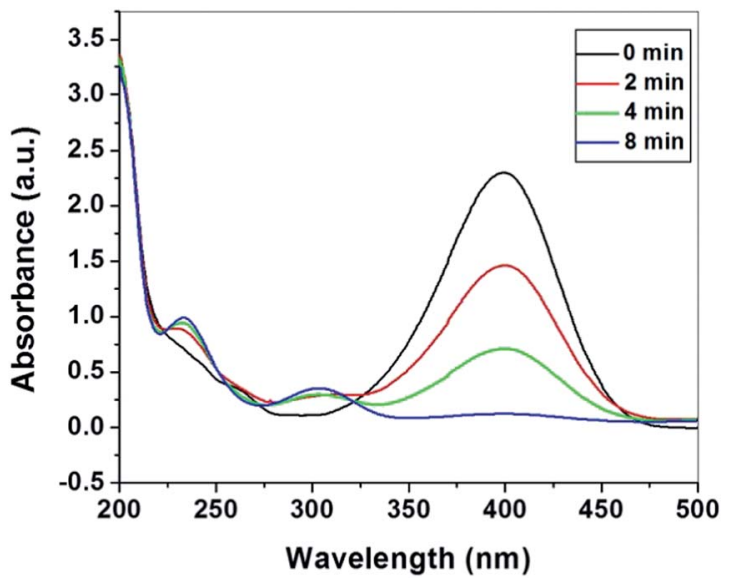

Fig. 5 UV-vis absorption spectra during the catalytic reduction of 4-NP by Au@TA-Fe catalyst.

reaction system. Also, no obvious change in the concentration of 4-NP can be detected after 30 min using TA-Fe complexes only as catalyst, demonstrating that the reduction of 4-NP by $\mathrm{NaBH}_{4}$ is solely catalyzed by Au NPs in the composites. Since excess $\mathrm{NaBH}_{4}$ was present and its concentration could be considered as a constant during the reaction period, the reduction process would be assessed as pseudo-first-order kinetics. The ratios of $C_{\mathrm{t}}$ to $C_{0}$ were obtained from the relative intensity ratios of the respective absorbances $\left(A_{\mathrm{t}} / A_{0}\right)$ at $400 \mathrm{~nm}$. The rate constants $k$ directly estimated from the slopes were $0.11 \mathrm{~min}^{-1}, 0.21 \mathrm{~min}^{-1}, 0.37 \mathrm{~min}^{-1}$, and $0.24 \mathrm{~min}^{-1}$ for bare $\mathrm{Au}$ NPs (synthesized through direct reduction of $\mathrm{HAuCl}_{4}$ by TA), Au@TA-Fe/1 cycle, Au@TA-Fe/2 cycles, and Au@TA-Fe/3 cycles, respectively (Fig. 6). As can be seen, Au NPs enclosed within TA-Fe coatings exhibited a higher catalytic performance than the bare Au NPs. This enhancement of catalytic activity may be attributed to the synergistic effect of Au NPs and the TA-Fe shells, similar to those of previous reports. Ke et al.

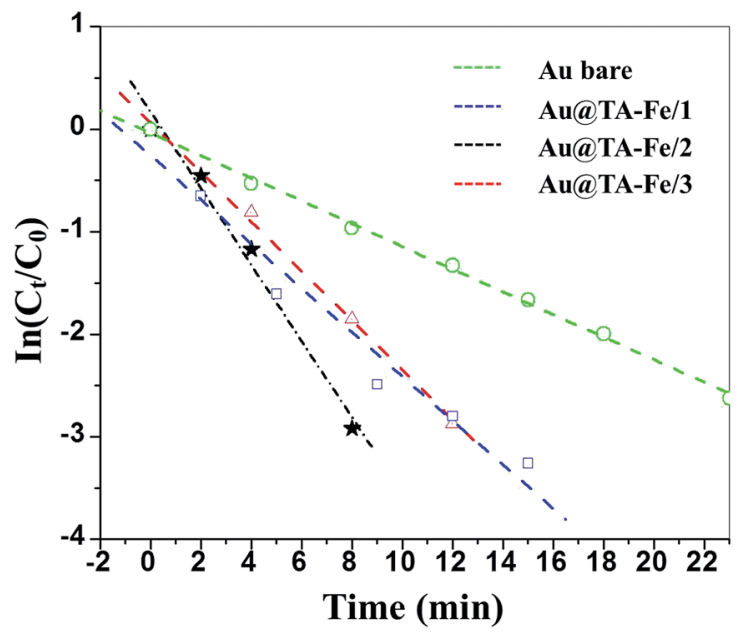

Fig. 6 Plots of $\ln \left(C_{t} / C_{0}\right)$ versus reaction time for bare Au NPs, Au@TAFe composites with 1,2 , and 3 assembly cycles. $C_{t}$ and $C_{0}$ are the concentrations of 4-NP at the beginning and at time $t$, respectively. 
prepared Au@MIL-100(Fe) core-shell nanocatalysts with improved catalytic performance and put this down to the $\pi-\pi$ stacking interaction between the aromatic rings of 4-NP and the organic linkers. ${ }^{\mathbf{1 4}}$ Here, the abundant aromatic rings of TA in the shell could also help to enrich 4-NP from the aqueous mixture through $\pi-\pi$ stacking interactions and thus give rise to a local high concentration of 4-NP and a superior catalytic efficiency of Au@TA-Fe toward 4-NP reduction. However, the over-thick coating (such as the 3-cycle assembly) resulted in a slight decrease of rate constant compared with a relatively thin coating (such as the 2-cycle assembly), which may be related to the increase in the diffusion path length of 4-NP. The exact reasons of the above phenomena still need more experimental or theoretical evidence. For fair comparison with the catalytic results reported by other groups, a normalizing $k_{\text {nor }}\left[k_{\text {nor }}=k / m\right.$, where $m$ is the Au content (umol) in the catalyst] is introduced because it can exclude the effect of metal loading on different catalysts according to Chen et al. ${ }^{32}$ Table $\mathrm{S} 1 \dagger$ summarizes the catalytic activity of different Au NP-based catalysts for 4-NP reduction. It was found that $k_{\text {nor }}\left(3.7 \mathrm{~min}^{-1} \mu \mathrm{mol}^{-1}\right)$ of the Au@TA-Fe is superior to many other Au NP- based catalysts.

We then investigated the $\mathrm{pH}$ effect on the catalytic activity of Au@TA-Fe composites under various buffer systems. From Fig. 7, the catalytic efficiency demonstrated a obvious dependence on $\mathrm{pH}$ and declined with decreasing $\mathrm{pH}$ value. This can be ascribed to the fact that the coordination between $\mathrm{Fe}^{3+}$ and TA is $\mathrm{pH}$ dependent. As reported, the catechol- $\mathrm{Fe}^{3+}$ coordination state changed from monospecies at $\mathrm{pH}<2$, bis- at $3<\mathrm{pH}<$ 6 , to tri- at $\mathrm{pH}>7$ (see Fig. $\mathrm{S} 5 \dagger$ ). ${ }^{21,33}$ Therefore, at low $\mathrm{pH}$, a rapid disassembly of the TA-Fe layer could be caused by the protonation of most hydroxyl groups, which may lead to the decrease in the enrichment capacity of the TA-Fe layer for 4-NP and further decrease the catalytic activity. In contrast, the TA-Fe layer would be stable and exhibit an enhancement in catalytic activity at relatively high $\mathrm{pH}$. This $\mathrm{pH}$-responsive character of the TA-Fe layer endows the presented method with great potential for assembly of "smart" functional materials for many applications, such as controlled drug delivery, biosensors, and bionanoreactors. ${ }^{34,35}$

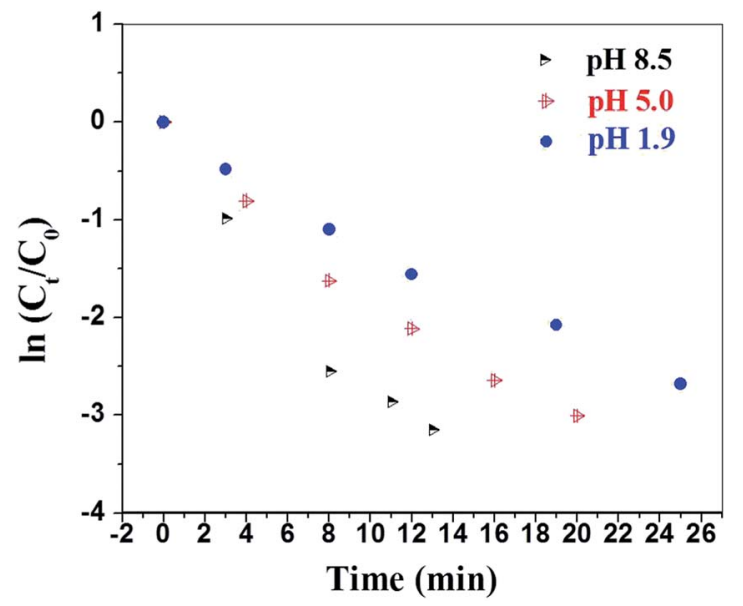

Fig. 7 Plots of $\ln \left(C_{t} / C_{0}\right)$ versus reaction time for the AuCTA-Fe catalyst at different $\mathrm{pH}$.
An important concern with respect to potential applications of catalysts is their reusability and stability in the operating process. The Au@TA-Fe catalysts were easily isolated from the reaction mixture with centrifugation after being used and reused in the next run. The result in Fig. S6† shows that it could be successfully recycled for four successive cycles with no significant loss of activity. Additionally, the TEM images of Au@TA-Fe catalysts after being used (Fig. S7†) revealed that the morphology remained almost unvaried, demonstrating a good stability of the as-prepared catalyst under the reaction conditions.

In summary, a novel Au@TA-Fe core-shell catalyst system was readily fabricated on the basis of PPs-metal coordination complexes through a low-cost, green and facile approach. The shells with $\mathrm{pH}$-responsive properties were easily formed on the surface of Au NPs by mixing different PPs with $\mathrm{Fe}^{3+}$ within an aqueous solution of Au NPs, and the thickness could be easily tuned by changing the number of assembly cycles. This nanocatalyst exhibited enhanced catalytic performance for the reduction of 4-NP to 4-AP with $\mathrm{NaBH}_{4}$ and good reusability compared with bare Au NPs. With great simplicity and operability, this innovative approach may be anticipated to hold significant promise for the synthesis of other core-shell nanosystems with unique characteristics for various applications.

\section{Acknowledgements}

We thank the National Basic Research Program of China (2011CB936001) and the National Natural Science Foundation of China (21277152, 21277002, and 21321004) for financial support.

\section{Notes and references}

1 A. Balanta, C. Godard and C. Claver, Chem. Soc. Rev., 2011, 40, 4973-4985.

2 A. Mohanty, N. Garg and R. C. Jin, Angew. Chem., Int. Ed., 2010, 49, 4962-4966.

3 S. Wu, J. Dzubiella, J. Kaiser, M. Drechsler, X. Guo, M. Ballauff and Y. Lu, Angew. Chem., Int. Ed., 2012, 51, 2229-2233.

4 M. Turner, V. B. Golovko, O. P. H. Vaughan, P. Abdulkin, A. Berenguer-Murcia, M. S. Tikhov, B. F. G. Johnson and R. M. Lambert, Nature, 2008, 454, 981-U931.

5 Z. J. Wang, Q. X. Zhang, D. Kuehner, A. Ivaska and L. Niu, Green Chem., 2008, 10, 907-909.

6 T. Zeng, X. L. Zhang, S. H. Wang, Y. R. Ma, H. Y. Niu and Y. Q. Cai, J. Mater. Chem. A, 2013, 1, 11641-11647.

7 J. Liu, H. Q. Yang, F. Kleitz, Z. G. Chen, T. Yang, E. Strounina, G. Q. Lu and S. Z. Qiao, Adv. Funct. Mater., 2012, 22, 591-599.

8 Y. Yang, J. Liu, X. B. Li, X. Liu and Q. H. Yang, Chem. Mater., 2011, 23, 3676-3684.

9 J. Luo, L. Wang, D. Mott, P. N. Njoki, Y. Lin, T. He, Z. Xu, B. N. Wanjana, I. I. S. Lim and C. J. Zhong, Adv. Mater., 2008, 20, 4342-4347.

10 J. M. Yan, X. B. Zhang, T. Akita, M. Haruta and Q. Xu, J. Am. Chem. Soc., 2010, 132, 5326-5327. 
11 Y. W. Cao, R. Jin and C. A. Mirkin, J. Am. Chem. Soc., 2001, 123, 7961-7962.

12 H. B. Li, W. J. Kang, B. J. Xi, Y. Yan, H. Y. Bi, Y. C. Zhu and Y. T. Qian, Carbon, 2010, 48, 464-469.

13 J. Lee, J. C. Park and H. Song, Adv. Mater., 2008, 20, 15231528.

14 F. Ke, J. F. Zhu, L. G. Qiu and X. Jiang, Chem. Commun., 2013, 49, 1267-1269.

15 C. F. Xiao, S. M. Chen, L. Y. Zhang, S. Q. Zhou and W. T. Wu, Chem. Commun., 2012, 48, 11751-11753.

16 T. S. Sileika, D. G. Barrett, R. Zhang, K. H. A. Lau and P. B. Messersmith, Angew. Chem., Int. Ed., 2013, 52, 1076610770.

17 S. Quideau, D. Deffieux, C. Douat-Casassus and L. Pouysegu, Angew. Chem., Int. Ed., 2011, 50, 586-621.

18 M. Andjelkovic, J. Van Camp, B. De Meulenaer, G. Depaemelaere, C. Socaciu, M. Verloo and R. Verhe, Food Chem., 2006, 98, 23-31.

19 Q. Ye, F. Zhou and W. M. Liu, Chem. Soc. Rev., 2011, 40, 4244-4258.

20 H. Xu, J. Nishida, W. Ma, H. Wu, M. Kobayashi, H. Otsuka and A. Takahara, ACS Macro Lett., 2012, 1, 457-460.

21 H. Ejima, J. J. Richardson, K. Liang, J. P. Best, M. P. van Koeverden, G. K. Such, J. W. Cui and F. Caruso, Science, 2013, 341, 154-157.

22 M. Scampicchio, J. Wang, A. J. Blasco, A. S. Arribas, S. Mannino and A. Escarpa, Anal. Chem., 2006, 78, 20602063.
23 Y. Lee and T. G. Park, Langmuir, 2011, 27, 2965-2971.

24 X. Huang, X. P. Liao and B. Shi, Green Chem., 2011, 13, 28012805.

25 S. Khokhar and R. K. O. Apenten, Food Chem., 2003, 81, 133140.

26 P. Mulvaney, Langmuir, 1996, 12, 788-800.

27 V. Kozlovskaya, E. Kharlampieva, I. Drachuk, D. Cheng and V. V. Tsukruk, Soft Matter, 2010, 6, 3596-3608.

28 S. E. Kwiatek, V. Desai, P. J. Moran and P. M. Natishan, J. Mater. Sci., 1997, 32, 3123-3128.

29 M. A. Rahim, H. Ejima, K. L. Cho, K. Kempe, M. Mullner, J. P. Best and F. Caruso, Chem. Mater., 2014, 26, 1645-1653.

30 Y. N. Zhou, X. H. Xing, Z. H. Liu, L. W. Cui, A. F. Yu, Q. Feng and H. J. Yang, Chemosphere, 2008, 72, 290-298.

31 T. Zeng, X. L. Zhang, Y. R. Ma, H. Y. Niu and Y. Q. Cai, J. Mater. Chem., 2012, 22, 18658-18663.

32 J. M. Zhang, G. Z. Chen, M. Chaker, F. Rosei and D. L. Ma, Appl. Catal., B, 2013, 132, 107-115.

33 N. Holten-Andersen, M. J. Harrington, H. Birkedal, B. P. Lee, P. B. Messersmith, K. Y. C. Lee and J. H. Waite, Proc. Natl. Acad. Sci. U. S. A., 2011, 108, 2651-2655.

34 M. Guo, Y. Yan, H. K. Zhang, H. S. Yan, Y. J. Cao, K. L. Liu, S. R. Wan, J. S. Huang and W. Yue, J. Mater. Chem., 2008, 18, 5104-5112.

35 J. L. Zhang, M. X. Zhang, K. J. Tang, F. Verpoort and T. L. Sun, Small, 2014, 10, 32-46. 